\title{
Autosomal dominant pseudohypoparathyroidism type Ib is associated with a heterozygous microdeletion that likely disrupts a putative imprinting control element of GNAS
}

\author{
Murat Bastepe, ${ }^{1}$ Leopold F. Fröhlich, ${ }^{1}$ Geoffrey N. Hendy, ${ }^{2}$ Olafur S. Indridason, ${ }^{3}$ \\ Robert G. Josse, ${ }^{4}$ Hiroyuki Koshiyama, ${ }^{5}$ Jarmo Körkkö, ${ }^{6}$ Jon M. Nakamoto, ${ }^{7}$ \\ Arlan L. Rosenbloom, ${ }^{8}$ Arnold H. Slyper, ${ }^{9}$ Toshitsugu Sugimoto, ${ }^{10}$ \\ Agathocles Tsatsoulis, ${ }^{11}$ John D. Crawford, ${ }^{12}$ and Harald Jüppner ${ }^{1,13}$ \\ ${ }^{1}$ Endocrine Unit, Department of Medicine, Massachusetts General Hospital and Harvard Medical School, \\ Boston, Massachusetts, USA \\ ${ }^{2}$ Calcium Research Laboratory, Royal Victoria Hospital, Departments of Medicine, Physiology, and Human Genetics, \\ McGill University, Montreal, Quebec, Canada \\ ${ }^{3}$ Renal Unit, Department of Medicine, Landspitali-University Hospital, Reykjavik, Iceland \\ ${ }^{4}$ Division of Endocrinology and Metabolism, St. Michael's Hospital, University of Toronto, Toronto, Ontario, Canada \\ ${ }^{5}$ Division of Diabetes and Endocrinology, Department of Medicine, Medical Research Institute, Kitano Hospital, and \\ Department of Diabetes and Clinical Nutrition, Kyoto University Graduate School of Medicine, Kyoto, Japan \\ ${ }^{6}$ Department of Clinical Genetics, Oulu University Hospital, Oulu, Finland \\ ${ }^{7}$ Division of Pediatric Endocrinology, Department of Pediatrics, David Geffen School of Medicine at UCLA, \\ Los Angeles, California, USA \\ ${ }^{8}$ Department of Pediatrics, Division of Endocrinology, University of Florida, Gainesville, Florida, USA \\ ${ }^{9}$ Department of Pediatrics, Medical College of Wisconsin, Milwaukee, Wisconsin, USA \\ ${ }^{10}$ Division of Endocrinology/Metabolism, Neurology and Hematology/Oncology Department of Clinical Molecular Medicine, \\ Kobe University Graduate School of Medicine, Kobe, Japan \\ ${ }^{11}$ Division of Endocrinology, Department of Medicine, University of Ioannina, Ioannina, Greece \\ ${ }^{12}$ Pediatric Endocrine Unit and \\ ${ }^{13}$ Pediatric Nephrology Unit, MassGeneral Hospital for Children, Massachusetts General Hospital and \\ Harvard Medical School, Boston, Massachusetts, USA
}

\begin{abstract}
Patients with pseudohypoparathyroidism type Ib (PHP-Ib) have hypocalcemia and hyperphosphatemia due to renal parathyroid hormone (PTH) resistance, but lack physical features of Albright hereditary osteodystrophy. PHP-Ib is thus distinct from PHP-Ia, which is caused by mutations in the GNAS exons encoding the $\mathrm{G}$ protein $\alpha$ subunit. However, an imprinted autosomal dominant form of PHP-Ib (ADPHP-Ib) has been mapped to a region of chromosome $20 \mathrm{q} 13.3$ containing GNAS. Furthermore, loss of methylation at a differentially methylated region (DMR) of this locus, exon A/B, has been observed thus far in all investigated sporadic PHP-Ib cases and the affected members of multiple AD-PHP-Ib kindreds. We now report that affected members and obligate gene carriers of 12 unrelated AD-PHP-Ib kindreds and four apparently sporadic PHP-Ib patients, but not healthy controls, have a heterozygous approximately $3-\mathrm{kb}$ microdeletion located approximately $220 \mathrm{~kb}$ centromeric of GNAS exon $\mathrm{A} / \mathrm{B}$. The deleted region, which is flanked by two direct repeats, includes three exons of STX16, the gene encoding syntaxin-16, for which no evidence of imprinting could be found. Affected individuals carrying the microdeletion show loss of exon A/B methylation but no epigenetic abnormalities at other GNAS DMRs. We therefore postulate that this microdeletion disrupts a putative cis-acting element required for methylation at exon $\mathrm{A} / \mathrm{B}$, and that this genetic defect underlies the renal PTH resistance in AD-PHP-Ib.
\end{abstract}

J. Clin. Invest. 112:1255-1263 (2003). doi:10.1172/JCI200319159.

Received for publication June 10, 2003, and accepted in revised form August 12, 2003.

Address correspondence to: Harald Jüppner, Endocrine Unit, Wellman 5, Massachusetts General Hospital, Boston, Massachusetts 02114, USA. Phone: (617) 726-3966; Fax: (617) 726-7543; E-mail: jueppner@helix.mgh.harvard.edu.

Jon M. Nakomoto's present address is: Division of Endocrinology, Quest Diagnostics Nichols Institute, San Juan Capistrano, California, USA. Conflict of interest: The authors have declared that no conflict of interest exists.

Nonstandard abbreviations used: pseudohypoparathyroidism (PHP); parathyroid hormone (PTH); PHP type Ia (PHP-Ia); Albright hereditary osteodystrophy (AHO); G protein $\alpha$ subunit (Gs $\alpha$ ); pseudo-PHP (PPHP); autosomal dominant PHP-Ib (AD-PHP-Ib); single nucleotide polymorphism (SNP); differentially methylated region (DMR).

\section{Introduction}

The most prominent findings in pseudohypoparathyroidism (PHP) are hypocalcemia, hyperphosphatemia, and elevated serum concentration of parathyroid hormone (PTH), which reflect end-organ resistance to the actions of this hormone $(1,2)$. In PHP type Ia (PHP-Ia), end-organ resistance is usually not limited to the PTH actions but can also affect other hormones, such as thyrotropin and gonadotropins $(1,2)$. Patients affected by PHP-Ia also have physical features of Albright hereditary osteodystrophy (AHO), including obesity, short stature, brachydactyly, and ectopic ossification. 
PHP-Ia is associated with inactivating defects of the stimulatory $G$ protein $\alpha$ subunit (Gs $\alpha$ ), which can be detected by an approximately $50 \%$ reduction in the level/activity of this protein in easily accessible cells such as erythrocytes $(1,2)$. These defects are caused by heterozygous mutations in one of the 13 exons of GNAS that encode Gsa (3).

In addition to Gs $\alpha$, GNAS gives rise to several other transcripts and protein products (Figure 1a). Transcripts encoding XLas and NESP55 are expressed mainly in neuroendocrine tissues and are derived from the paternal or maternal GNAS allele, respectively (4-9). The A/B (also referred to as exon $1 \mathrm{~A}$ or $1^{\prime}$ ) and the antisense transcripts, which are presumed to be untranslated, show ubiquitous expression and are both derived from the paternal allele (10-13). Furthermore, the promoters of these transcripts are differentially methylated, and in each case transcription takes place from the nonmethylated promoter. In contrast, the promoter driving expression of Gs $\alpha$ lacks differential methylation and is biallelically active in most tissues $(8,9,14,15)$. However, through as-yet-undefined mechanisms, paternal Gs $\alpha$ expression is silenced in certain cells, including the cells of the renal proximal tubules, the pituitary gland, the thyroid gland, and the gonads (16-19).

The same GNAS mutations resulting in inactive Gs $\alpha$ mutants are also found in a variant of PHP-Ia termed pseudo-PHP (PPHP), which is characterized by the presence of $\mathrm{AHO}$ without hormone resistance $(1,2)$. Patients with PHP-Ia and PPHP are typically found within the same kindred, but not within the same sibship, and it is now known that inheritance of a Gs $\alpha$ mutation from a mother affected by either PHP-Ia or PPHP leads to PHP-Ia, i.e., AHO with hormone resistance, while inheritance of the same mutation from a father affected by either disorder leads to PPHP, i.e., AHO without hormone resistance (20, 21). Thus, in a given PHP-Ia/PPHP kindred, the type of disorder that develops in the offspring depends on the gender of the parent transmitting the mutation, rather than the type of disorder in that parent (Figure 1b). This parent-of-origin-specific mode of inheritance in PHP-Ia/PPHP kindreds is consistent with recent findings indicating that Gs $\alpha$ expression occurs predominantly from the maternal allele in some tissues or cells (16-19).

PHP-Ib is another variant of PHP in which end-organ resistance appears to be limited primarily to the actions of PTH in the renal cortex, thus leading to hypocalcemia and hyperphosphatemia. Patients with PHP-Ib do not present with AHO, and GNAS mutations leading to reduced Gs $\alpha$ activity have been excluded in this disorder $(1,2)$. However, an autosomal dominant form of PHP-Ib (AD-PHP-Ib) has been

\section{Figure 1}

genetically linked to a region on $20 \mathrm{q} 13.3$ that includes GNAS (22). In addition, similar to the hormone-resistance phenotype in PHP-Ia/PPHP kindreds, PTH resistance in AD-PHP-Ib has been shown to develop only in offspring of female obligate carriers, i.e., offspring inheriting the genetic defect from male obligate carriers do not have PTH resistance (see Figure 1b) $(22,23)$. Moreover, all sporadic and familial PHP-Ib cases investigated thus far show a loss of methylation at the maternal GNAS exon $\mathrm{A} / \mathrm{B}$ and biallelic expression of $\mathrm{A} / \mathrm{B}$ transcripts $(23,24)$. These findings suggested that AD-PHP-Ib is caused by a mutation or mutations in a cis-acting element of GNAS that controls exon A/B methylation and that the loss of methylation at this site leads to a profound reduction or a complete lack of Gs $\alpha$ expression in the renal proximal tubules (and possibly a few other tissues), where this signaling protein is predominantly derived from the maternal allele (16). Recently, genetic studies and direct sequence analyses strongly suggested that the genetic mutation responsible for the exon $\mathrm{A} / \mathrm{B}$ methylation defect and the resulting PTH resistance is located more than $50 \mathrm{~kb}$ upstream of exon $\mathrm{A} / \mathrm{B}$, thus implicating a long-range regulatory element in the pathogenesis of AD-PHP-Ib (23).

Through further genetic analyses of different AD-PHPIb kindreds, we have now been able to significantly reduce the size of the critical interval and to identify within this interval a heterozygous microdeletion that segregates with the disease in multiple kindreds. The same microdeletion was also found in some apparently sporadic PHP-Ib cases, but not in healthy unrelated controls.

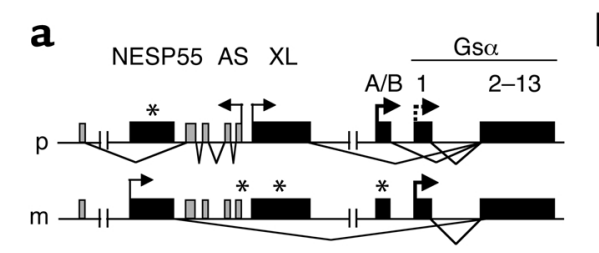

b

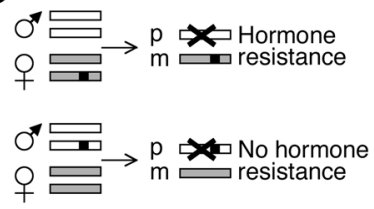

The GNAS locus and parent-of-origin-specific inheritance of hormone resistance. (a) GNAS gives rise, besides Gs $\alpha$, to multiple other transcripts including NESP55, $\mathrm{XL \alpha s}$, the antisense (AS) and $\mathrm{A} / \mathrm{B}$ (also referred to as $1 \mathrm{~A}$ or $1^{\prime}$ ), all of which show differential methylation (asterisks) in their promoters and are expressed exclusively from the non-methylated paternal $(p)$ or maternal $(m)$ allele. Exons that lead to transcripts in the sense (right-pointing arrow) and antisense (left-pointing arrow) directions are depicted as black and gray boxes, respectively, and the splice patterns are indicated. Note that the promoter giving rise to the Gs $\alpha$ transcript, encoded by exons 1 through 13, does not show differential methylation. However, although expression is biallelic in most tissues, Gs $\alpha$ transcription from the paternal GNAS allele is proposed to be silenced in a limited number of cells (dotted arrow), including those in renal proximal tubules. (b) In kindreds with PHP-la and PPHP, as well as in those with AD-PHP-lb, the genetic defect (black squares) leads to hormone resistance only if it is inherited from a female obligate carrier of the GNAS mutation. On the other hand, hormone resistance does not develop if the genetic defect is inherited from a male obligate carrier. Each black square indicates the mutation leading to PHP-la/PPHP or AD-PHP-lb. Paternal and maternal alleles of the GNAS locus are depicted by white and gray rectangles, respectively. X's indicate the silencing of paternal Gs $\alpha$ expression that occurs in a small number of cells, including those of the renal proximal tubules. 


\section{Methods}

Patients with PHP-Ib. Most of the investigated AD-PHPIb cases were previously reported $(22,23,25)$. Biochemical features of the probands in the previously noninvestigated AD-PHP-Ib families are provided in Table 1 . The study was approved by the Subcommittee on Human Studies of Massachusetts General Hospital, and informed consent was obtained from all subjects.

Genotype analysis. Most markers on chromosome $20 \mathrm{q} 13.3$ that were analyzed in this study and the conditions for their amplification by PCR have been previously described $(22,23,26)$. Microsatellite marker 261P9-CA1 was amplified with the following primers: 5'-ACATGTGTGTGCACCTGTCC-3' (forward) and 5'GGCTTTTCTCAAAGCACCAC- $3^{\prime}$ (reverse). The C/T single nucleotide polymorphism (SNP) 261P9-15,686 was amplified with the following primers: 5'ATGTTTCCCCTTGATTGTGC-3' (forward) and $5^{\prime}$ GCCTGCCTACCTAAATCTT-3' (reverse). MslI, whose recognition sequence is introduced by the thymidine nucleotide, was used to determine the parental origin of each allele. The $\mathrm{C}$ allele gives rise to 664-bp and 426-bp fragments, and the T allele to 473-bp, 191-bp, and 426-bp fragments. PCR conditions were as described previously (23).

Detection of the 3-kb microdeletion by Southern blot analysis. (The approximately $3-\mathrm{kb}$ microdeletion will be referred to hereafter as a 3-kb microdeletion.) Genomic DNA was digested with AvrII or ClaI and subsequently separated on a $0.8 \%$ agarose gel before transfer onto nitrocellulose. Blots were hybridized with PCR-generated ${ }^{32} \mathrm{P}$-labeled probes corresponding to nucleotides 2,163-3,906 and 15,213-16,303 of AL139349, respectively. Hybridization and washing conditions were as described previously (22).

Detection of the 3- $k b$ microdeletion by PCR. The deleted region was amplified with the following forward and reverse primers: $5^{\prime}$-TTGGCAGATAACTGCTGTGG-3' and 5'-CGAGGCATGGgaAgagaATA-3'. The Expand longtemplate PCR system was used with Buffer 3 (Roche Molecular Biochemicals, Indianapolis, Indiana, USA). Cycling conditions included one cycle of $94^{\circ} \mathrm{C}$ for $2 \mathrm{~min}$ utes; ten cycles of $94^{\circ} \mathrm{C}$ for 10 seconds, $58^{\circ} \mathrm{C}$ for $30 \mathrm{sec}$ - onds, and $68^{\circ} \mathrm{C}$ for 4 minutes; 25 cycles of $94^{\circ} \mathrm{C}$ for 10 seconds, $58^{\circ} \mathrm{C}$ for 30 seconds, and $68^{\circ} \mathrm{C}$ for 4 minutes, with an additional 20 seconds at $68^{\circ} \mathrm{C}$ after each cycle; and a final extension step at $68^{\circ} \mathrm{C}$ for 7 minutes. Based on the location of the primers, the product corresponded to nucleotides 3,606-7,951 of AL139349, thus yielding a fragment of approximately $4.3 \mathrm{~kb}$ from the wildtype allele and of approximately $1.3 \mathrm{~kb}$ from the mutant allele. These fragments were detected by $0.7 \%$ agarose gel electrophoresis followed by ethidium bromide staining.

Sequence analysis of the region deleted in AD-PHP-Ib and the coding STX16 exons. Three overlapping PCRs were used to amplify the 3-kb region from genomic DNA of patients who lack the microdeletion. PCR was also used to amplify individual STX16 exons from genomic DNA of an affected individual from kindred W (W-III/12). PCR products were directly sequenced using nested sequencing primers as previously described (23). Obtained sequences were compared with the sequence of AL139349, except for the sequence from STX16 exon 1 , which was compared with the sequence of AL050327. Primer sequences are available upon request.

Methylation analysis of GNAS. To assess differential methylation, Southern blots of genomic DNA digested with methylation-sensitive enzymes were performed as described (23). Alternatively, or in combination, differential methylation was assessed by direct sequence and restriction enzymatic analyses of PCR products amplified from bisulfite-treated genomic DNA (bisulfite PCR analysis). Conditions for bisulfite treatment and primer sequences for amplification of the exon $\mathrm{A} / \mathrm{B}$ region were as previously described (27). The amplicon from the latter region was sequenced directly, and also digested with either FauI or Hinfl to determine cytosine-to-thymidine conversion. The following forward and reverse primers were used to amplify the exon NESP55 region from bisulfite-treated DNA: 5'-GAGGATAAAGATTTAAGGGATTT-3' and $5^{\prime}$-CTCAAACTCCCCAATTTAAC-3' for primary PCR, and $5^{\prime}$-GAAGGAGTTTAAGGAGGAGAAGTAG-3' and $5^{\prime}$ CCATAAAAACAAAAAAAATCTAAAC- 3 ' for nested PCR. The amplicon was directly sequenced and also digested with AciI. The following primers were used to amplify the exon XL region from bisulfite-treated DNA: 5'-GGTAGTT-

Table 1

Information on index cases in previously noninvestigated AD-PHP-Ib kindreds

\begin{tabular}{|c|c|c|c|c|c|c|c|}
\hline Kindred & $\mathrm{T} 2$ & 1 & K & $\mathrm{H} 1$ & $\mathrm{H} 2$ & T3 & $\mathrm{H} 3$ \\
\hline Age at presentation (yr) & 26 & 30 & 8 & 39 & 8 & 16 & 14 \\
\hline Additional affected members & 3 & 2 & 3 & 2 & $0^{\mathrm{A}}$ & $0^{\mathrm{A}}$ & 0 \\
\hline PTH $(p g / m l)$ & 653 & 208 & 381 & $1530^{\mathrm{B}}$ & $185^{C}$ & 247 & 168 \\
\hline (normal range) & $12-72$ & $10-55$ & $10-55$ & $290-850$ & $10-65$ & $10-65$ & $20-65$ \\
\hline Calcium (mmol/l) & 1.95 & 1.14 & 1.41 & 1.80 & 2.10 & 1.10 & 2.03 \\
\hline (normal range) & $2.05-2.65$ & $2.2-2.60$ & $2.15-2.70$ & $2.05-2.65$ & $2.15-2.70$ & $2.05-2.65$ & $2.13-2.63$ \\
\hline Phosphate $(\mathrm{mmol} / \mathrm{l})$ & 1.42 & 2.08 & 3.18 & 1.15 & 2.26 & 3.42 & 2.00 \\
\hline (normal range) & $0.8-1.6$ & $0.8-1.6$ & $1.2-1.8$ & $0.8-1.6$ & $1.2-1.8$ & $0.8-1.6$ & $0.8-1.6$ \\
\hline
\end{tabular}

AMaternal DNA samples, which were available for the index cases in kindreds $\mathrm{H} 2$ and T3, also showed the 3-kb microdeletion, indicating that these mothers are unaffected obligate carriers. DNA samples from the parents of the index case in kindred $\mathrm{H} 3$ were unavailable. ${ }^{\mathrm{B} P T H}$ was measured by a radioimmunoassay specific for the C-terminal portion of PTH, rather than by two-site immunometric assay as for the other cases. CNot the value at presentation, but measured after the patient had discontinued her treatment for 3 weeks. 


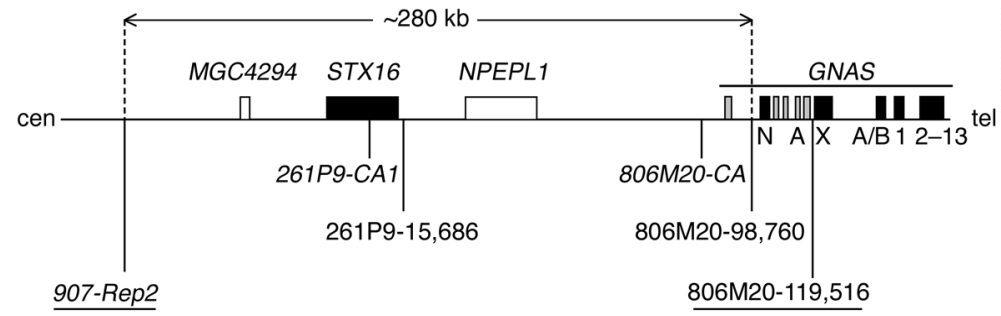

\section{Figure 2}

The AD-PHP-Ib critical interval. The AD-PHP-Ib genetic interval was determined by recombination events in kindreds $W$ (centromeric [cen]) and $F$ (telomeric [tel]). Boundary markers are underlined; microsatellites are in italics. While the distance between 907-Rep2 and $806 \mathrm{M} 20-119516$ is about $300 \mathrm{~kb}$, the region between $806 \mathrm{M} 20-98760$ and $806 \mathrm{M} 20-119516$ has been previously excluded through direct sequence analysis (23). Thus, the critical interval comprises approximately $280 \mathrm{~kb}$. Note that 261P9-CA1 is a dinucleotide repeat located within intron 6 of STX16. Known and predicted genes within the linked interval are depicted as filled or open boxes, respectively (note that individual exons are not shown). For GNAS, exons are shown with black (sense) and gray (antisense) boxes ( $N$, exon NESP55; $A$, exons encoding an antisense transcript; $X$, exon XL; A/B, exon A/B; 1-13, exons encoding Gs $\alpha$ ). MGC4294 (National Center for Biotechnology Information locus ID: 79160) and NPEPL1 (locus ID: 79716) are predicted genes. Note that the centromeric boundary of the AD-PHP-Ib locus was previously defined at D20S149 $(22,23)$. However, the daughter of W-II/9 in kindred W (23) was shown to have a loss of exon A/B methylation, making the recombination event in $\mathrm{W}-\mathrm{II} / 9$ informative, which redefined the centromeric boundary at 907-Rep2.

TATTTTAAGAGGTTGTTAGATTT-3' and 5'-AAAAAAATACTTTTCCTCCCTCC-3' for primary PCR, and $5^{\prime}$ GGGTAGTAGTTTTTGGATGGAGAT-3' and $5^{\prime}$-CATCTCTACTACTTCCTCCTCAACTAAA-3' for nested PCR. The amplicon was directly sequenced or digested with SalI. Methylation analyses of the deleted region and STX16 promoter. Bisulfite PCR analysis was used for assessment of differential methylation at $15 \mathrm{CPG}$ dinucleotides located within STX16 exon 4. Through the use of leukocyte genomic DNA from an affected member of kindred $\mathrm{F}$ (F$\mathrm{IV} / 47$ ) who inherited the $3-\mathrm{kb}$ deletion from her mother, or from an unaffected obligate carrier of the same kindred (F-III/34) who inherited the same deletion from her father (22), separate analysis of the paternal and maternal alleles could be performed. The analysis was also performed on DNA from a normal control and the previously reported patient with paternal uniparental isodisomy of chromosome $20 \mathrm{q}(28)$. The first PCR used the following forward and reverse primers: $5^{\prime}$ GATTGAGTGAATAGAGTTTTTTATTTTT- $3^{\prime}$ and $5^{\prime}$-ATTCCAAACCAAAATTTCACATAAA- 3 '; the nested PCR used the following forward and reverse primers: 5 -GTGGTAGTGTTAGTAGAGGGGTTTTT- ${ }^{\prime}$ and $5^{\prime}$-AACATATTCAAAAACTAACAATAAAAAATA- 3 '. The nested amplicon, which corresponded to nucleotides 4,501-4,848 of AL139349, was sequenced directly or after subcloning into pCR4-TOPO vector from Invitrogen Corp. (Carlsbad, California, USA). Restriction endonucleases Hinfl or Acil were also used to digest the amplicons for assessment of bisulfitemediated cytosine-to-thymidine conversion. Methylation of $\mathrm{CpG}$ dinucleotides was also investigated in two different regions within the $\mathrm{CPG}$ island spanning the putative STX16 promoter. Analysis was performed through the use of genomic DNA samples from a normal control and the patient with paternal uniparental isodisomy of chromosome 20q (28). Primary PCR for one of these regions used 5'-TTGTTTTAATGGATGTTTTATATTAATTTT- ${ }^{\prime}$ and $5^{\prime}$-AAACAAAAACTCCAAACAACCTAAC- $3^{\prime}$ as forward and reverse primers, respectively. Nested PCR used 5'-GGGTTAGGTTTTTGGTTTTAAGTTT- $3^{\prime}$ and $5^{\prime}$-AAAAATCTCTATTCCCTACCTAAACC- $3^{\prime}$. The nested product (nucleotides 108,821-109,103 of AL050327) was either sequenced directly or digested with either $H h a \mathrm{I}$ or HpyCH4IV. Primary PCR for the second region used 5'-TGGGGATTATGTTTTTAATTTGATT- ${ }^{\prime}$ and $5^{\prime}$-AAAAACCCCAACCAAACAAC- $3^{\prime}$ as forward and reverse primers, respectively. Nested PCR for the second region used $5^{\prime}$ GTAGTGTGGGGATAAATTTTTTTT-3' and $5^{\prime}$-AACATCCATTAAAACAAATAACAAAATAC- $3^{\prime}$. The nested product (nucleotides 108,044-108,382 of AL050327) was either sequenced directly or digested with Hinfl. In all reactions to amplify the bisulfite-treated DNA, HotMaster Taq from Eppendorf North America Inc. (Westbury, New York, USA) was used. Conditions included one cycle of $94^{\circ} \mathrm{C}$ for 2 minutes; 40 cycles (primary) or 25 cycles (nested) of $94^{\circ} \mathrm{C}$ for 45 seconds, $54^{\circ} \mathrm{C}$ for 45 seconds, and $65^{\circ} \mathrm{C}$ for 2 minutes; and one cycle of $65^{\circ} \mathrm{C}$ for 10 minutes.

Nucleotide sequence analyses and comparisons. To identify the regions conserved in mouse and rat, "percent identity plots" for human (AL139349-GenBank) versus mouse (ENSMUSG00000027522-Ensembl Gene ID) or human versus rat (ENSRNOG00000005281) genomic DNA were generated by MultiPipMaker (http://bio.cse.psu.edu) (29); no sequences were masked except for the dinucleotide repeat in intron 6 (261P9-CA1). Dot blots were generated by PipMaker (http://bio.cse.psu.edu). CpG content analysis was performed by CPGPLOT, which uses the following criteria for $\mathrm{CpG}$ island identification (30): (i) observed/expected $\mathrm{CPG}>0.6$; (ii) \% $(\mathrm{C}+\mathrm{G})>50$; and (iii) length > $200 \mathrm{bp}$.

\section{Results}

The centromeric boundary of the genetic interval for ADPHP-Ib has been previously defined at marker D20S149 $(22,23)$. Analysis of genomic DNA from a previously unstudied child in kindred W (23), W-III/12, revealed a loss of methylation at GNAS exon A/B (data not shown). This finding made the recombination event in her mother (W-II/9) informative, thereby redefining the centromeric boundary of the AD-PHP-Ib locus at marker 907Rep2 (Figure 2). Thus the critical interval was reduced to approximately $280 \mathrm{~kb}$, which included STX16, the gene encoding syntaxin-16 $(31,32)$; two predicted genes, 
a

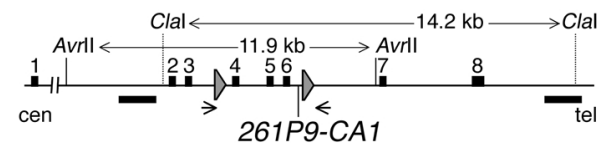

b

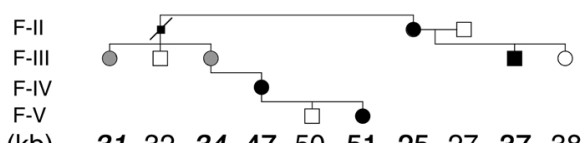

(kb) $\quad \begin{array}{llllllllll}31 & 32 & 34 & 47 & 50 & 51 & 25 & 27 & 37 & 38\end{array}$

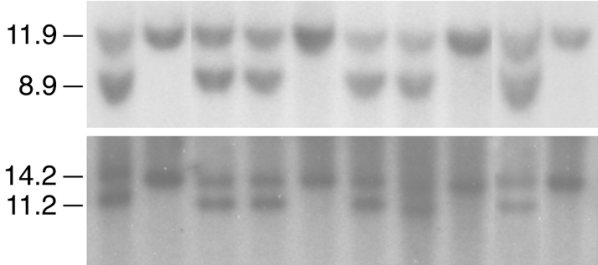

C

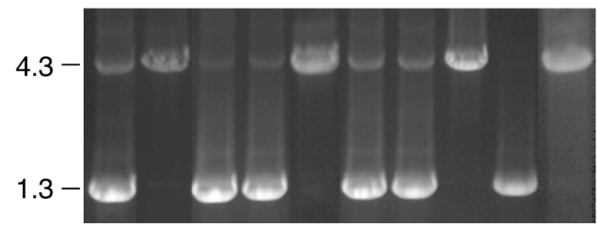

Figure 3

Identification of the 3-kb microdeletion in kindred F. (a) Genomic structure of the region surrounding the microdeletion. Filled boxes and connecting lines depict STX16 exons and introns, respectively. Horizontal bars represent the probes used in Southern blot analysis. Arrowheads indicate the location of the two 391. bp direct repeats (nucleotides 3,786-4,176 and nucleotides 6,764-7,154 of AL139349). Arrows indicate the approximate positions of the PCR primers used for amplification of the wildtype and mutant alleles. (b) Southern blot analysis of genomic DNA from selected members of kindred $F(22)$. Affected individuals, filled symbols and bold identification numbers. Healthy individuals, open symbols and plain numbers. Unaffected obligate gene carrier, gray circles and bold italic numbers. Genomic DNA digested with either Avrll (top) or Clal (bottom) was separated on $0.8 \%$ agarose and transferred onto nitrocellulose before hybridization with the radiolabeled probe. (c) PCR amplification of the wild-type $(\sim 4.3 \mathrm{~kb})$ and mutant $(\sim 1.3 \mathrm{~kb})$ alleles from genomic DNA of members of kindred $F(22)$. Nucleotide sequence analysis of the 1.3-kb PCR product derived from the mutant allele revealed that 2,978 bp were deleted (based on AL139349 sequence), although this number varies due to polymorphisms including the dinucleotide repeat polymorphism 261P9-CA1 (see Supplemental Table 1; www.jci.org/cgi/content/full/112/8/1255/DC1).

and no abnormalities at other differentially methylated regions (DMRs) of GNAS (Table 2). These 11 kindreds, who reside in several different countries and are of different ethnic and racial backgrounds, included seven previously published familial cases $(22,23,25)$ and four recently identified kindreds with at least three affected
MGC4294 and NPEPL1; and a small centromeric portion of GNAS. To search for a mutation within this region, a cosmid library was constructed using genomic DNA from F-III/37, an affected individual of kindred F (22). For determining the parental origin of isolated cosmids, novel and known polymorphic markers that would be informative for this individual were identified. During the course of these studies, an allelic loss was detected at marker 261P9-CA1 in affected individuals and unaffected obligate diseasegene carriers of kindred $\mathrm{F}$ (data not shown). A 391-bp sequence just telomeric of 261P9-CA1 was, with the exception of two base pairs, directly repeated 2,978 bp (based on AL139349) further centromeric (Figure 3a). The allelic loss, in combination with these direct repeats, suggested the presence of a heterozygous disease-associated microdeletion, which was confirmed by Southern blot analyses using genomic DNA digested with either AvrII or ClaI (Figure 3b). Wild-type and mutant alleles could also be amplified by PCR, thus allowing confirmation of the deletion and providing a simple assay for mutation detection (Figure 3c). Sequence analysis of the mutant $1.3-\mathrm{kb}$ PCR product showed that one of the two repeats and the intervening nucleotides were deleted, thus removing a 3 -kb region including STX16 exons 4, 5, and 6 (see Figure 3a).

Identical heterozygous microdeletions were also identified in affected members and obligate carriers, but not in the healthy members, of 11 additional unrelated AD-PHP-Ib kindreds that show loss of A/B methylation

\section{Table 2}

Epigenetic and genetic characteristics of the investigated PHP-Ib kindreds

\begin{tabular}{|c|c|c|c|c|c|}
\hline Kindred ${ }^{A}$ & $\begin{array}{l}\text { Loss of } \mathrm{A} / \mathrm{B} \\
\text { methylation }\end{array}$ & $\begin{array}{c}\text { Methylation } \\
\text { defects at } \\
\text { other GNAS } \\
\text { DMRs }\end{array}$ & $\begin{array}{c}3-k b \\
\text { deletion }\end{array}$ & $\begin{array}{c}\text { Parental } \\
\text { origin of } \\
\text { deletion } \\
\text { in patients }\end{array}$ & Reference \\
\hline $\mathrm{F}$ & + & - & + & Mat & 22,23 \\
\hline P & + & - & + & Mat & 22 \\
\hline $\mathrm{D}$ & + & - & + & Mat & 22 \\
\hline T1 & + & - & + & Mat & 22 \\
\hline S1 & + & - & + & Mat & 23 \\
\hline Y1 & + & - & + & Mat & 23 \\
\hline $\mathrm{E}$ & + & - & + & Mat & 23,25 \\
\hline A & + & - & + & Mat & 25 \\
\hline $\mathrm{T} 2$ & + & - & + & Mat & This report ${ }^{B}$ \\
\hline I & + & - & + & Mat & This report ${ }^{\mathrm{B}}$ \\
\hline K & + & - & + & Mat & This report $\mathrm{B}^{\mathrm{B}}$ \\
\hline $\mathrm{H} 1$ & + & - & + & ND & This report ${ }^{B}$ \\
\hline $\mathrm{H}_{2} \mathrm{C}$ & + & - & + & Mat & This report ${ }^{B}$ \\
\hline $\mathrm{T}^{\mathrm{C}}$ & + & - & + & Mat & This report $\mathrm{B}^{\mathrm{B}}$ \\
\hline $\mathrm{B}^{\mathrm{C}}$ & + & - & + & ND & 25 \\
\hline $\mathrm{H} 3^{\mathrm{C}}$ & + & - & + & ND & This report $\mathrm{B}^{\mathrm{B}}$ \\
\hline W & + & - & - & NA & 23 \\
\hline Y2 & + & + & - & NA & 23 \\
\hline
\end{tabular}

The index cases in families E, A, and B were previously reported as patients 1,2 , and 8 respectively (25). ${ }^{A}$ Note that these unrelated kindreds were from different countries and different ethnic and racial backgrounds. ${ }^{B}$ Biochemical features of the index cases of new kindreds reported in this study are given in Table 1. CThese were apparently sporadic PHP-Ib cases, as there were no additional affected family members. Mat, maternal; ND, not determined due to unavailability of parental DNA samples. 


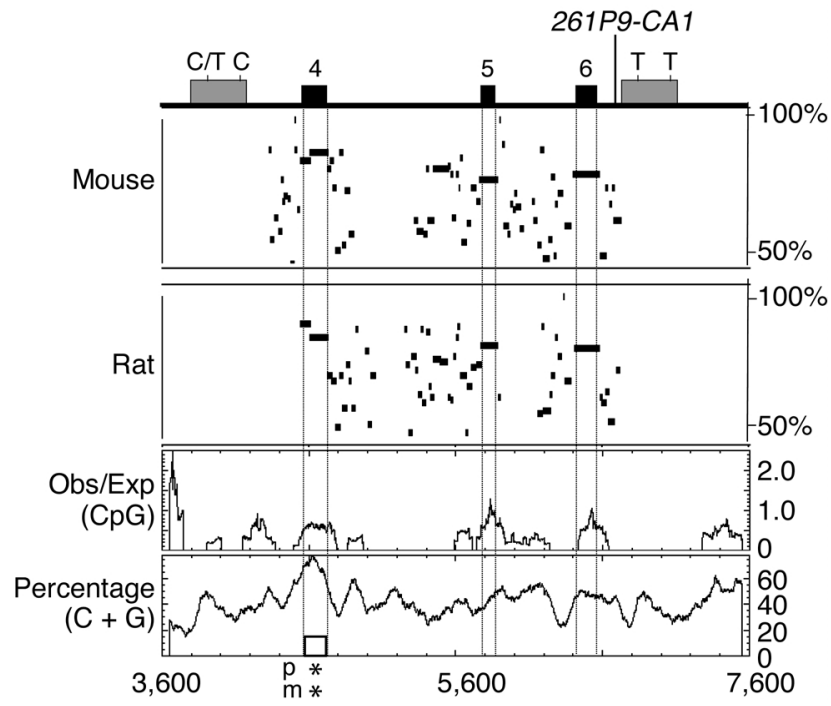

Figure 4

Genomic structure of the human chromosomal region containing the AD-PHP-lb microdeletion. The illustrated region, extending from nucleotides 3,600 to 7,600 of AL139349, contains STX16 exons 4-6 (black boxes), which are flanked by almost-identical 391-bp direct repeats (gray boxes); note that only two nucleotides differ between the two repeat sequences and that nucleotide 3,959 is a polymorphic C/T (see Supplemental Table 1; www.jci.org/cgi/content/full/112/ $8 / 1255 / D C 1)$. The approximate location of the dinucleotide repeat marker 261P9-CA1 is indicated. Horizontal bars represent uninterrupted sequence stretches showing more than $50 \%$ identity between human and mouse or human and rat. Note that the longest stretch of nucleotide sequence conservation, besides STX16 exons 4-6, is observed in a 98-bp segment located within intron 4 (nucleotides $5,460-5,557$ of AL139349). Dot blots confirmed the location of this segment in intron 4 of mouse and rat Stx 16 (not shown); sequences homologous to this region could not be found in GenBank. The 391bp repeats are not present in the corresponding regions of mouse and rat genomes. No CpG islands are found in the deleted region. However, a 140-bp segment within exon 4 (white box; nucleotides 4,572-4,711 of AL139349) has an observed/expected (Obs/Exp) $\mathrm{CpG}>0.6$ and $\%(C+G)>50$; note that the corresponding regions in mouse and rat do not meet these criteria. Bisulfite PCR analysis indicated that all $15 \mathrm{CpG}$ dinucleotides in this region were methylated (asterisks) on the maternal $(m)$ and paternal $(p)$ alleles, i.e., there was no evidence for differential, parent-specific methylation.

individuals. For each affected individual, the microdeletion was inherited from the mother, which is consistent with the maternal-specific inheritance of PTH resistance in $\mathrm{AD}$-PHP-Ib $(22,23)$. Genomic DNA from 115 healthy controls, which included unrelated spouses from each kindred, did not have the microdeletion, as determined by PCR. The 4.3-kb wild-type product could be amplified from each sample, whereas the $1.3-\mathrm{kb}$ mutant PCR product could not be amplified from any of the samples (data not shown). Taken together, these findings indicated a strong genetic association between AD-PHP-Ib and the identified mutation.

We also screened 16 apparently sporadic PHP-Ib cases. Four of the cases, who showed loss of exon A/B methylation only, had the same microdeletion identified in AD-PHP-Ib kindreds (see Table 2). Analysis of parental
DNA samples, which were available for two of the four cases with the deletion, showed that the mutations were inherited from the mothers, who were clinically and biochemically unaffected. These two patients thus represented previously unrecognized familial cases.

In contrast with these findings, the $3-\mathrm{kb}$ microdeletion was not found in kindreds $\mathrm{Y} 2$ and $\mathrm{W}$, in whom genetic analyses and the mode of inheritance did not argue against linkage to the GNAS locus (23). While kindred Y2 showed a broad methylation abnormality involving multiple DMRs of GNAS, kindred W exhibited a loss-of-methylation defect only at exon A/B and was therefore investigated further. However, direct sequence analysis of genomic DNA from affected individual W-III/12 excluded a heterozygous small deletion or a point mutation within the $3-\mathrm{kb}$ region, along with mutations in the remaining coding STX16 exons (data not shown). Likewise, analysis of genomic DNA from the 12 sporadic PHP-Ib cases who were found to exhibit epigenetic defects involving not only exon A/B but also exons NESP55 and XL did not have the $3-\mathrm{kb}$ microdeletion. Furthermore, nucleotide sequence analyses of these individuals revealed a total of 18 polymorphisms but did not identify any smaller deletions or point mutations within the $3-\mathrm{kb}$ region that is deleted in most AD-PHP-Ib kindreds (see Supplemental Table 1; www.jci.org/cgi/content/full/112/8/1255/DC1).

The deleted region is flanked by two direct repeats and includes three exons of STX16. Note that the 391-bp repeats differ by two nucleotides: nucleotides 3,959 and 4,147 in the first repeat are cytosine nucleotides, while the corresponding nucleotides in the second repeat (nucleotides 6,937 and 7,125) are thymidine nucleotides. However, nucleotide 3,959 is polymorphic and can be either cytosine or thymidine. The repeat remaining in the mutant allele had a thymidine at this position in 14 of the 16 cases with the microdeletion. All 16 had thymidine nucleotides at the other (telomeric) site.

Comparison of the nucleotide sequence with the regions of conserved synteny in mouse and rat genomes indicate that the deleted exons of STX16 are well conserved and that the introns are similar in length (Figure 4). In addition, there are a number of conserved intronic sequences, including a stretch of 98 bp located within intron 4 . The direct repeats, on the other hand, are not present in mouse and rat. Based on analysis of the nucleotide content within the deleted region, guanine and cytosine nucleotides comprise $43 \%$ of the entire sequence, and the overall CpG dinucleotide content is $1.4 \%$. No CpG islands are present in the deleted region, but a small cluster of $\mathrm{CpG}$ dinucleotides is located within STX16 exon 4 (see Figure 4). To determine whether this region was subject to differential methylation, we investigated the methylation status of $\mathrm{CPG}$ dinucleotides in this cluster through analysis of bisulfite-treated leukocyte DNA. This analysis revealed that both parental alleles were methylated, indicating the absence of differential methylation (data not shown). 


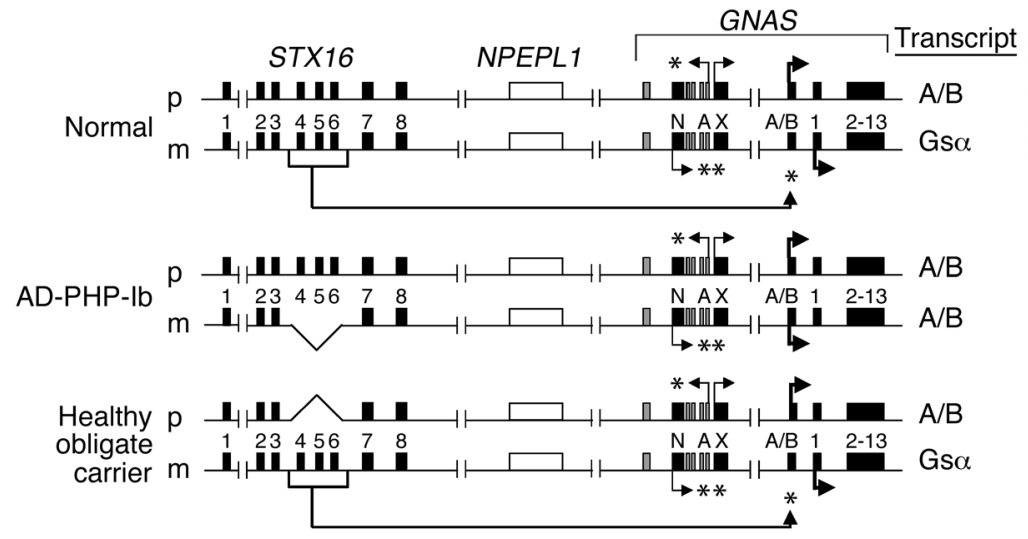

\section{Figure 5}

Possible molecular mechanism underlying the marked loss of Gs $\alpha$ expression in the renal proximal tubule in AD-PHP-Ib. Maternal inheritance of the microdeletion is associated with loss of methylation at exon $A / B$ and no epigenetic abnormalities at the NESP55 and XL DMRs, while paternal inheritance of the same deletion is not associated with methylation defects. We thus postulate that the identified microdeletion, which includes STX16 exons 4-6, disrupts a putative cis-acting element regulating the exon $A / B$ methylation imprint. It has been suggested that in the renal proximal tubules, a lack of exon $\mathrm{A} / \mathrm{B}$ methylation and/or active transcription of $\mathrm{A} / \mathrm{B}$ mRNA, both of which are normally seen on the paternal GNAS allele, mediate, in cis, the silencing of Gs $\alpha$ transcription (24). Therefore, the maternal loss of exon A/B methylation in AD-PHP-lb is predicted to cause a marked reduction in Gs $\alpha$ expression levels in that tissue, thereby leading to resistance to PTH (and perhaps to other hormones), whose action is mediated by the PTH/PTHrP receptor, a predominantly Gs $\alpha$-coupled receptor (43). Black and gray boxes depict exons of STX16 and GNAS in the sense or antisense direction, respectively. White boxes, NPEPL1; asterisks, CpC methylation; arrows, direction of active transcription.

\section{Discussion}

Through analysis of informative markers within the AD-PHP-Ib critical interval, we detected an allelic loss in affected individuals and unaffected obligate carriers of kindred F, the largest AD-PHP-Ib kindred that defined the telomeric boundary of the genetic locus. This finding led to the identification of a heterozygous microdeletion of approximately $3 \mathrm{~kb}$ located approximately $220 \mathrm{~kb}$ centromeric of GNAS exon A/B. The same mutation was also found in each of the affected members and unaffected carriers of 11 additional kindreds, in whom the epigenetic abnormality in the affected individuals is limited to the exon A/B DMR. Thus, all but one of our familial cases who show loss of exon A/B methylation and no abnormalities at other GNAS DMRs carry the 3-kb microdeletion. Furthermore, four PHP-Ib patients who were initially thought to represent sporadic cases were also shown to carry the same mutation. Consistent with this finding, epigenetic abnormalities in these patients were limited to exon A/B. In two of these cases, we determined that the deletion was inherited from the unaffected mother. On the other hand, none of the sporadic or familial PHPIb patients demonstrating broad GNAS methylation abnormalities were found to have the $3-\mathrm{kb}$ microdeletion. It thus appears that the identified microdeletion is specific for and frequently found (16 of 17 cases) in those PHP-Ib individuals who show an imprinted auto- somal dominant mode of inheritance (i.e., AD-PHP-Ib) and a methylation defect limited to exon A/B.

The heterozygous deletion removes three of the eight exons encoding syntaxin-16, a ubiquitously expressed member of the syntaxin family of SNARE proteins $(31,32)$. Syntaxin-16 is localized mainly in the Golgi apparatus in the cell, where it is thought to be involved in intracellular trafficking $(31,32)$. Although the AD-PHP-Ib mutation is predicted to result in a nonfunctional protein with an in-frame deletion of 133 amino acids, syntaxin-16 haploinsufficiency appears unlikely to explain the loss of exon A/B methylation and PTH resistance in ADPHP-Ib. First, individuals who inherited the disrupted copy of STX16 paternally, i.e., unaffected obligate carriers, exhibit proper GNAS methylation and are clinically and biochemically normal. Second, renal PTH resistance can develop despite the presence of two intact copies of STX16, as shown in a patient with paternal uniparental isodisomy of $20 \mathrm{q}$ (28). Moreover, there are no reports suggesting that STX16 is imprinted, and we could not find any evidence of differential methylation within the $\mathrm{CpG}$ island that contains the putative promoter of this gene (data not shown). Thus, involvement of STX16 and/or its protein product in the molecular pathogenesis of AD-PHP-Ib appears unlikely, although oogenesis-specific mechanisms could be postulated.

Instead, based on the nature of the epigenetic defect observed in patients carrying the $3-\mathrm{kb}$ microdeletion, we propose that the deletion disrupts a long-range cis-acting element regulating methylation of GNAS exon A/B (Figure 5). Methylation of exon A/B and its promoter is established in the female germline and maintained on the maternal allele through pre- and postimplantation development and is thus considered to represent an "imprint mark" (10). In contrast, it has not been clearly determined whether differential methylation of the promoters of NESP55, XLas, and AS transcripts are also established during gametogenesis (10). It is therefore possible that the cis-acting element disrupted by the deletion identified in AD-PHP-Ib is involved in the establishment and/or maintenance of the exon $\mathrm{A} / \mathrm{B}$ imprint in the female germline rather than its maintenance in somatic cells. For example, this element could be required for proper interaction between the exon $\mathrm{A} / \mathrm{B}$ region and one or more DNA methyltransferases in the female germline. Further investigations are thus required to determine the role of the deleted region in regulating exon $\mathrm{A} / \mathrm{B}$ methylation.

Imprinting control elements that show long-range effects have been defined for other imprinted loci in human and mouse (33), and microdeletions disrupting 
these regulatory elements have been identified in human disorders other than AD-PHP-Ib $(34,35)$. Unlike most imprinting control elements, the deleted region in $\mathrm{AD}$-PHP-Ib has a low $\mathrm{CPG}$ content and shows no evidence for differential methylation (see Figure 4). However, based on these features, it resembles the imprinting center disrupted in certain patients with Angelman syndrome (36-38). While no sequence homology could be found between the deleted regions in Angelman syndrome and AD-PHP-Ib, some of the evolutionarily conserved intronic sequences in the region deleted in $\mathrm{AD}$-PHP-Ib may represent regulatory elements required for exon A/B methylation.

The two direct repeats flanking the AD-PHP-Ib microdeletion consist of AT-rich, MIR, and MER46A interspersed repeat elements, as well as intervening unique sequences, and do not seem to be repeated as a whole elsewhere in the genome. Repetitive DNA sequences have been located near deletion breakpoints in other inherited disorders. While highly repetitive Alu elements appear to be most commonly involved in these deletions (39), low-copy repeats that flank disease-associated deletions have also been reported in certain disorders, such as DiGeorge syndrome (40) and PraderWilli/Angelman syndrome (41). As suggested previously for these other disorders, a mechanism involving homologous recombination between the flanking repeats may have led to the deletion in AD-PHP-Ib founders. A deletion generated through such a mechanism is predicted to occur more frequently than other types of mutations (such as random genomic rearrangements and single nucleotide changes), indicating a preferred mechanism for causing a mutation. This prediction suggests that the $3-\mathrm{kb}$ microdeletion has arisen independently in each kindred. Consistent with this hypothesis, we found the mutation to be associated in different AD-PHP-Ib kindreds with different alleles of a C/T SNP (261P9-15,686; see Figure 2) located approximately $10 \mathrm{~kb}$ to the telomere ( $\mathrm{C}$ in two kindreds and $\mathrm{T}$ in eight kindreds), strongly suggesting that it has occurred on at least two different haplotype backgrounds. This hypothesis is further supported by having the $3-\mathrm{kb}$ microdeletion in multiple kindreds of divergent ethnic and racial backgrounds. In fact, the microdeletion was the only mutation identified in our collection of PHP-Ib patients, which is consistent with the predicted high frequency of this type of mutation. Nevertheless, investigation of additional patients may reveal other mutations within the deleted region or in adjacent DNA sequences, and these may help delineate more precisely the putative regulatory element involved in the molecular pathogenesis of AD-PHP-Ib and possibly other forms of PHP-Ib.

Two of the previously published PHP-Ib kindreds with an apparent autosomal trait did not carry the 3$\mathrm{kb}$ microdeletion (see Table 2). Kindred Y2 has broad epigenetic abnormalities affecting several DMRs of GNAS (23), and therefore the absence of the identified microdeletion in this kindred was not entirely unexpected. For kindred W, however, the epigenetic changes are indistinguishable from those observed in other families with AD-PHP-Ib (23). Furthermore, two-point lod scores for markers in this region strongly suggest that the genetic defect in kindred $\mathrm{W}$ is located close to GNAS (lod score of 2.5 at D20S171 [ $\theta=0$ ], which is identical to the maximal theoretical lod score). However, affected individuals of this kindred do not have other mutations within the $3-\mathrm{kb}$ region that is deleted in most AD-PHP-Ib kindreds, or mutations in the remaining coding STX16 exons (data not shown). It thus appears likely that methylation of exon $\mathrm{A} / \mathrm{B}$ requires an additional cis-acting element that is mutated in kindred W.

We observed methylation abnormalities at multiple GNAS DMRs in 12 sporadic cases, as well as in two PHP-Ib kindreds with multiple affected individuals other than kindred Y2 (M. Bastepe and H. Jüppner, unpublished observations). Owing to the small number of DNA samples currently available, linkage of the genetic defect in these kindreds to the previously established AD-PHP-Ib locus has not been confirmed. However, already available genetic data do not argue against linkage to GNAS. Furthermore, at least for kindred Y2, methylation of a distinct DMR on chromosome $20 \mathrm{q}$ that involves NNAT, the gene encoding neuronatin (42), is preserved (23). These findings suggest that the genetic defect in this kindred, and perhaps in other sporadic or familial forms of PHP-Ib with broad GNAS methylation abnormalities, also resides in proximity to GNAS. Hence, there may be yet another putative cis-acting element involved in regulating methylation at all DMRs of this gene, rather than exon $\mathrm{A} / \mathrm{B}$ alone.

In summary, we have identified a microdeletion associated with AD-PHP-Ib in multiple kindreds. The microdeletion is located within STX16 approximately 220-kb centromeric of GNAS exon A/B, i.e., upstream of the DMR at which AD-PHP-Ib patients show loss of methylation. Additional investigations will be necessary to elucidate the molecular mechanisms resulting in the epigenetic and biochemical abnormalities observed in AD-PHP-Ib and its variants. Insights from these studies will not only clarify the different biological roles of GNAS, but are also likely to enhance our understanding of other imprinted genes. Furthermore, new information may be gained regarding the tissue- and cell-specific silencing of the ubiquitously expressed Gs $\alpha$, and such studies could lead to the discovery of additional mechanisms that may help explain Gso-dependent end-organ responsiveness to a large variety of different hormones.

\section{Acknowledgments}

We thank Henry M. Kronenberg, Stephen M. Krane, Brian Seed, and Robert C. Gensure for critical review of the manuscript. We also thank the members of different PHP-Ib kindreds who participated in this study. This work was supported by grants from NIH NIDDK (RO1 46718-10 to H. Jüppner and KO1 DK-06297301A1 to M. Bastepe) and the Canadian Institutes of Health Research (to G.N. Hendy). 
1. Levine, M.A. 2002. Pseudohypoparathyroidism. In Principles of bone biology. J.P. Bilezikian, L.G. Raisz, and G.A. Rodan, editors. Academic Press. New York, New York, USA. 1137-1163.

2. Weinstein, L.S., Yu, S., Warner, D.R., and Liu, J. 2001. Endocrine manifestations of stimulatory $G$ protein alpha-subunit mutations and the role of genomic imprinting. Endocr. Rev. 22:675-705.

3. Kozasa, T., Itoh, H., Tsukamoto, T., and Kaziro, Y. 1988. Isolation and characterization of the human Gsa gene. Proc. Natl. Acad. Sci. U. S. A. 85:2081-2085.

4. Kehlenbach, R.H., Matthey, J., and Huttner, W.B. 1994. XLas is a new type of G protein [erratum 1995, 375:253]. Nature. 372:804-809.

5. Pasolli, H., Klemke, M., Kehlenbach, R., Wang, Y., and Huttner, W. 2000. Characterization of the extra-large $G$ protein alpha-subunit XLalphas. I. Tissue distribution and subcellular localization. J. Biol. Chem. 275:33622-33632.

6. Pasolli, H., and Huttner, W. 2001. Expression of the extra-large G protein alpha-subunit XLalphas in neuroepithelial cells and young neurons during development of the rat nervous system. Neurosci. Lett. 301:119-122.

7. Ischia, R., et al. 1997. Molecular cloning and characterization of NESP55, a novel chromogranin-like precursor of a peptide with 5-HT1B receptor antagonist activity. J. Biol. Chem. 272:11657-11662.

8. Hayward, B., et al. 1998. The human GNAS1 gene is imprinted and encodes distinct paternally and biallelically expressed $\mathrm{G}$ proteins. Proc. Natl. Acad. Sci. U. S. A. 95:10038-10043.

9. Hayward, B.E., Moran, V., Strain, L., and Bonthron, D.T. 1998. Bidirectional imprinting of a single gene: GNAS1 encodes maternally, paternally, and biallelically derived proteins. Proc. Natl. Acad. Sci. U. S. A. 95:15475-15480.

10. Liu, J., Yu, S., Litman, D., Chen, W., and Weinstein, L. 2000. Identification of a methylation imprint mark within the mouse Gnas locus. Mol. Cell. Biol. 20:5808-5817.

11. Swaroop, A., Agarwal, N., Gruen, J.R., Bick, D., and Weissman, S.M. 1991 Differential expression of novel Gs alpha signal transduction protein cDNA species. Nucleic Acids Res. 19:4725-4729.

12. Ishikawa, Y., Bianchi, C., Nadal-Ginard, B., and Homcy, C.J. 1990. Alternative promoter and $5^{\prime}$ exon generate a novel $\mathrm{G}_{s} \alpha$ mRNA. J. Biol. Chem. 265:8458-8462.

13. Hayward, B., and Bonthron, D. 2000. An imprinted antisense transcript at the human GNAS1 locus. Hum. Mol. Genet. 9:835-841.

14. Zheng, H., Radeva, G., McCann, J.A., Hendy, G.N., and Goodyer, C.G. 2001. Gas transcripts are biallelically expressed in the human kidney cortex: implications for pseudohypoparathyroidism type Ib. J. Clin. Endocrinol. Metab. 86:4627-4629.

15. Campbell, R., Gosden, C.M., and Bonthron, D.T. 1994. Parental origin of transcription from the human GNAS1 gene. J. Med. Genet. 31:607-614.

16. Yu, S., et al. 1998. Variable and tissue-specific hormone resistance in heterotrimeric $\mathrm{G}_{s}$ protein $\alpha$-subunit $\left(\mathrm{G}_{s} \alpha\right)$ knockout mice is due to tissuespecific imprinting of the $\mathrm{G}_{\mathrm{s}} \alpha$ gene. Proc. Natl. Acad. Sci. U. S. A 95:8715-8720.

17. Hayward, B., et al. 2001. Imprinting of the G(s)alpha gene GNAS1 in the pathogenesis of acromegaly. J. Clin. Invest. 107:R31-R36.

18. Mantovani, G., Ballare, E., Giammona, E., Beck-Peccoz, P., and Spada, A. 2002. The Gsalpha gene: predominant maternal origin of transcription in human thyroid gland and gonads. J. Clin. Endocrinol. Metab. 87:4736-4740.

19. Germain-Lee, E.L., et al. 2002. Paternal imprinting of Galpha(s) in the human thyroid as the basis of TSH resistance in pseudohypoparathyroidism type 1a. Biochem. Biophys. Res. Commun. 296:67-72.

20. Davies, A.J., and Hughes, H.E. 1993. Imprinting in Albright's hereditary osteodystrophy. J. Med. Genet. 30:101-103.

21. Nakamoto, J.M., Sandstrom, A.T., Brickman, A.S., Christenson, R.A., and Van Dop, C. 1998. Pseudohypoparathyroidism type Ia from maternal but not paternal transmission of a Gs $\alpha$ gene mutation. Am. J. Med. Genet. 77:261-267.
22. Jüppner, H., et al. 1998. The gene responsible for pseudohypoparathyroidism type Ib is paternally imprinted and maps in four unrelated kindreds to chromosome 20q13.3. Proc. Natl. Acad. Sci. U. S. A. 95:11798-11803.

23. Bastepe, M., et al. 2001. Positional dissociation between the genetic mutation responsible for pseudohypoparathyroidism type $\mathrm{Ib}$ and the associated methylation defect at exon A/B: evidence for a long-range regulatory element within the imprinted GNAS1 locus. Hum. Mol. Genet. 10:1231-1241.

24. Liu, J., et al. 2000. A GNAS1 imprinting defect in pseudohypoparathyroidism type IB. J. Clin. Invest. 106:1167-1174.

25. Schipani, E., et al. 1995. Pseudohypoparathyroidism type Ib is not caused by mutations in the coding exons of the human parathyroid hormone (PTH)/PTH-related peptide receptor gene. J. Clin. Endocrinol. Metab. 80:1611-1621.

26. Bastepe, M., Pincus, J., and Jüppner, H. 1999. Two frequent tetranucleotide repeat polymorphisms between VAPB and STX16 on chromosome 20q13. Mol. Cell. Probes. 14:449-451.

27. Judson, H., Hayward, B.E., Sheridan, E., and Bonthron, D.T. 2002. A global disorder of imprinting in the human female germ line. Nature. 416:539-542.

28. Bastepe, M., Lane, A.H., and Jüppner, H. 2001. Paternal uniparental isodisomy of chromosome $20 \mathrm{q}$ (patUPD20q) -and the resulting changes in GNAS1 methylation-as a plausible cause of pseudohypoparathyroidism. Am. J. Hum. Genet. 68:1283-1289.

29. Schwartz, S., et al. 2000. PipMaker-a web server for aligning two genomic DNA sequences. Genome Res. 10:577-586.

30. Larsen, F., Gundersen, G., Lopez, R., and Prydz, H. 1992. CpG islands as gene markers in the human genome. Genomics. 13:1095-1107.

31. Simonsen, A., Bremnes, B., Ronning, E., Aasland, R., and Stenmark, H. 1998. Syntaxin-16, a putative Golgi t-SNARE. Eur. J. Cell Biol. 75:223-231.

32. Tang, B.L., Low, D.Y., Lee, S.S., Tan, A.E., and Hong, W. 1998. Molecular cloning and localization of human syntaxin 16, a member of the syntaxin family of SNARE proteins. Biochem. Biophys. Res. Commun. 242:673-679.

33. Reik, W., and Walter, J. 2001. Genomic imprinting: parental influence on the genome. Nat. Rev. Genet. 2:21-32.

34. Nicholls, R.D., and Knepper, J.L. 2001. Genome organization, function, and imprinting in Prader-Willi and Angelman syndromes. Annu. Rev. Genomics Hum. Genet. 2:153-175.

35. Cassidy, S.B., Dykens, E., and Williams, C.A. 2000. Prader-Willi and Angelman syndromes: sister imprinted disorders. Am. J. Med. Genet. 97:136-146.

36. Buiting, K., et al. 1995. Inherited microdeletions in the Angelman and Prader-Willi syndromes define an imprinting centre on human chromosome 15. Nat. Genet. 9:395-400.

37. Buiting, K., Lich, C., Cottrell, S., Barnicoat, A., and Horsthemke, B. 1999. A 5-kb imprinting center deletion in a family with Angelman syndrome reduces the shortest region of deletion overlap to 880 bp. Hum. Genet. 105:665-666.

38. Perk, J., et al. 2002. The imprinting mechanism of the PraderWilli/Angelman regional control center. EMBO J. 21:5807-5814.

39. Deininger, P.L., and Batzer, M.A. 1999. Alu repeats and human disease. Mol. Genet. Metab. 67:183-193.

40. Edelmann, L., et al. 1999. A common molecular basis for rearrangement disorders on chromosome 22q11. Hum. Mol. Genet. 8:1157-1167.

41. Amos-Landgraf, J.M., et al. 1999. Chromosome breakage in the PraderWilli and Angelman syndromes involves recombination between large, transcribed repeats at proximal and distal breakpoints. Am. J. Hum. Genet. 65:370-386.

42. Dou, D., and Joseph, R. 1996. Cloning of human neuronatin gene and its localization to chromosome-20q 11.2-12: the deduced protein is a novel "proteolipid". Brain Res. 723:8-22.

43. Jüppner, H., et al. 1991. A G protein-linked receptor for parathyroid hormone and parathyroid hormone-related peptide. Science. 254:1024-1026. 